\title{
Densidade energética de briquetes de biomassa lignocelulósica e sua relação com os parâmetros de briquetagem
}

\author{
Frederico de Souza1* ${ }^{1 *}$ Ailton Teixeira do Vale ${ }^{2}$ \\ ${ }^{1}$ Instituto Federal de Educação, Ciência e Tecnologia de Brasília, SubCentro Leste, Complexo Boca da Mata, Lote 1, CEP 72304-300, Samambaia, DF, Brasil \\ Universidade de Brasília, Asa Norte, CEP 70910-900, Brasília, DF, Brasil
}

*Autor correspondente:
frederico.souza@ifb.edu.br

Termos para indexação:

Bioenergia

Compactação de biomassa

Resíduos agroflorestais

Index terms:

Bioenergy

Biomass compression

Agroforestry waste

Histórico do artigo:

Recebido em 08/09/2015

Aprovado em 16/09/2016

Publicado em 30/12/2016

doi: 10.4336/2016.pfb.36.88.879
Resumo - A densidade energética é uma das principais propriedades que definem a qualidade do briquete, pois resume as características químicas, as condições de umidade e a quantidade de calor do produto final em uma única variável. Para analisála, foram confeccionados seis briquetes por tratamento (pinus, tauari, cumaru, casca de arroz, bagaço de cana e torta de pinhão manso) em uma prensa briquetadeira Lippel modelo LB-32 com $40 \mathrm{~g}$ de biomassa cada e 5 min de prensagem. Os parâmetros de briquetagem foram: duas temperaturas $\left(130\right.$ e $\left.200^{\circ} \mathrm{C}\right)$, duas pressões de compactação $\left(80\right.$ e $\left.120 \mathrm{kgf} \mathrm{cm}^{-2}\right)$ e dois tamanhos de partículas (grossas - retidas em peneira 35 mesh; finas - não retidas a 35 mesh). A densidade energética foi obtida multiplicando-se o poder calorífico útil pela densidade aparente. Adicionalmente, realizou-se a análise química imediata. Os resíduos florestais tiveram o carbono fixo $9,0 \%$ superior e cinzas $2,3 \%$ inferior, comparados aos resíduos agrícolas. A densidade aparente dos briquetes variou de 1,088 a $1,305 \mathrm{~g} \mathrm{~cm}^{-3}$ e a densidade energética entre 3,11-4,67 $\mathrm{Gcal} \mathrm{m}^{-3}$. Os briquetes de cumaru foram considerados de melhor qualidade, com densidades aparente e energética de $1,23 \mathrm{~g} \mathrm{~cm}^{-3}$ e 4,61 Gcal m${ }^{-3}$, respectivamente. Concluiu-se que o efeito da temperatura favoreceu a qualidade do briquete, superando os efeitos da pressão de compactação e tamanho da partícula.

\section{Energy density of lignocellulosic biomass briquettes and its relationship with briquetting parameters}

\begin{abstract}
Energy density is one of the main properties that define the quality of briquette as it summarizes chemical characteristics, moisture conditions and amount of heat of the final product in only one variable. We made six briquettes per treatment (pine, tauari, cumaru, rice husk, sugarcane bagasse and jatropha press cake) in a briquetting press Lippel LB-32 model with $40 \mathrm{~g}$ biomass each and $5 \mathrm{~min}$ of pressing. Briquetting parameters evaluated were: two temperatures $\left(130\right.$ and $\left.200{ }^{\circ} \mathrm{C}\right)$, two compacting pressures ( 80 to $120 \mathrm{kgf} \mathrm{cm}^{-2}$ ) and two particle sizes (thick - retained on 35 mesh sieve, thin - passing through 35 mesh sieve). The energy density was obtained by multiplying the useful heat content by bulk density. Additionally, we carried out chemical analysis. Forest residues had fixed carbon 9.0\% higher and ash $2.3 \%$ lower when compared to agricultural waste. Briquettes bulk density ranged from 1.088 to $1.305 \mathrm{~g} \mathrm{~cm}^{-3}$ and energy density between 3.11 and $4.67 \mathrm{Gcal} \mathrm{m}^{-3}$. Cumaru briquettes were considered of better quality, presenting $1.23 \mathrm{~g} \mathrm{~cm}^{-3}$ and $4.61 \mathrm{Gcal} \mathrm{m}^{-3}$ respectively for bulk and energy densities. It was concluded that the effect of temperature favors briquettes quality, overcoming effects of compression pressure and particle size.
\end{abstract}




\section{Introdução}

Briquetes são produtos compactados de resíduos agroflorestais ou de outra biomassa lignocelulósica, consolidados a partir da ação de temperaturas acima de $120^{\circ} \mathrm{C}$ e pressão acima de $80 \mathrm{kgf} \mathrm{cm}^{-2}$ para formar blocos de forma definida e de volume reduzido, facilitando principalmente o armazenamento, transporte e o uso da biomassa (Kaliyan \& Morey, 2009). O produto formado possui a finalidade de liberação de energia após a combustão, para processos industriais em fornos e caldeiras (Quirino \& Brito, 1991).

Segundo Pinheiro et al. (2005), as indústrias agroflorestais, por exemplo a sucroalcooleira, alimentícia e madeireira, são importantes setores da economia que produzem resíduos com grande potencial de aproveitamento energético no Brasil, dentre eles: bagaço de cana, palhas, cascas, serragem e maravalha que podem ser convertidos em material com maior valor comercial e com maior conteúdo energético por unidade de volume. Os resíduos gerados para o bagaço de cana no período de 2009/2010 foi cerca de 166 mil t, enquanto que os resíduos de madeira gerados no mesmo período foram aproximadamente $50 \mathrm{mil} \mathrm{t}$ (Souza et al., 2012a). Em geral, esses resíduos apresentam grande variedade de formas, densidades, granulometrias e teores de umidade, que influenciam diretamente no processo de compactação da biomassa para a manufatura dos briquetes.

Pesquisas recentes indicam grande preocupação na avaliação dos parâmetros da briquetagem e sua influência na qualidade do produto final, determinada pela densidade energética. As propriedades que são comumente avaliadas são: tamanho da partícula, temperatura e pressão de compactação e tempo de compactação e de armazenamento (Brand et al., 2009; Kaliyan \& Morey, 2009; Protásio et al., 2011; Karunanithy et al., 2012; Olakeji \& Enweremadu, 2012; Quirino et al., 2012).

Segundo Pinheiro et al. (2005), a densidade energética (DE) ou quantidade de calor por unidade de volume (Gcal $\mathrm{m}^{-3}$ ou GJ $\mathrm{m}^{-3}$ ) é a propriedade que avalia o desempenho da biomassa como combustível, pois resume as características físicas e químicas (constituição química, poder calorífico e cinzas) e é definida como o produto entre o poder calorífico e a densidade. Para Quirino \& Brito (1991), a partir da densidade aparente do briquete é que se determina a quantidade de energia ou calor acumulada neste material com volume definido.

Neste sentido, é possível observar diversas formas de cálculo desta característica, em que mais frequentemente é utilizado o poder calorífico superior e a densidade aparente unitária (Furtado et al., 2010; Protásio et al., 2011), cujo resultado é a quantidade de energia da biomassa seca por unidade de volume. Também pode ser utilizado o produto do poder calorífico útil pela densidade aparente, densidade do granel ou densidade do estéreo na mesma umidade. Contudo, deve-se atentar para a interpretação dos resultados da densidade energética, quando da comparação entre biomassas. Portanto, a forma que expressa a condição mais próxima da realidade referente ao conteúdo energético para os briquetes é o uso do poder calorífico útil e a densidade aparente (Brito, 1993; Vale et al., 2007).

Este estudo teve como objetivo, determinar a influência dos parâmetros de briquetagem, entre eles os fatores inerentes à biomassa e ao processo de fabricação, como o tamanho da partícula, pressão e temperatura, nas densidades aparente e energética de briquetes confeccionados por seis biomassas agroflorestais.

\section{Material e métodos}

Para este estudo foram selecionadas seis diferentes biomassas, incluindo três materiais oriundos da obtenção de madeira serrada (desdobramento de pranchas em tábuas) e três oriundos de processos industriais de produção de alimentos, etanol e biodiesel.

As biomassas de origem florestal selecionadas foram: pinus (Pinus spp.), tauari (Couratari guianensis Aubl.) e cumaru (Dipteryx odorata Aubl. Willd.), devido à maior disponibilidade no mercado madeireiro na região de Brasília, DF, e por abrangerem uma ordem crescente de densidade básica. A madeira de pinus apresenta densidade básica de aproximadamente $0,37 \mathrm{~g} \mathrm{~cm}^{-3}$ (Melo et al., 2013), o tauari cerca de $0,60 \mathrm{~g} \mathrm{~cm}^{-3}$ (Quirino et al., 2004) e o cumaru cerca de $1,09 \mathrm{~g} \mathrm{~cm}^{-3}$ (Quirino et al., 2004; Couto \& Barcellos, 2011). As amostras foram adquiridas em uma madeireira na forma de pranchas, com dimensões aproximadas de $200 \times 20$ x 4,5 cm (comprimento $\mathrm{x}$ largura $\mathrm{x}$ espessura). As espécies de madeira foram identificadas macroscopicamente por meio da comparação com as amostras da Xiloteca do Laboratório de Produtos Florestais do Serviço Florestal Brasileiro (Index Xylarium FPBw). 
Para as biomassas de origem agrícola selecionouse a casca de arroz (Oriza sativa L.), bagaço-de-cana (Saccharum officinarum) e torta de pinhão-manso (Jatropha curcas L.), por representarem atividades agrícolas importantes para as regiões brasileiras CentroOeste e Sudeste.

A casca de arroz, resíduo da produção de alimentos, foi obtida em uma empresa de materiais agrícolas na região de Brasília, DF, enquanto que a torta de pinhão manso, resíduo gerado pelo processamento (esmagamento) da semente do pinhão-manso para a produção de biocombustível, foi adquirido no município de Janaúba, MG, e o o bagaço-de-cana foi proveniente de uma usina de produção de etanol combustível, no município de Lençóis Paulista, SP.

Inicialmente, os materiais de origem florestal foram desdobrados em plaina desengrossadeira de marcenaria, reduzindo toda a prancha de madeira em maravalha (ou cepilho). A transformação da madeira em maravalha teve como finalidade a redução das partículas para que se fizesse a moagem. No caso dos resíduos agrícolas, não foram necessárias etapas prévias de preparação.

As maravalhas e os resíduos agrícolas de cada espécie foram moídos separadamente em moinho de facas tipo Willey. Posteriormente, fez-se a classificação com peneira de abertura da malha de 35 mesh (aproximadamente $0,355 \mathrm{~mm}$ ) na mesa vibratória, resultando em dois diferentes materiais moídos: 1- partículas retidas até 35 mesh (partícula grossa); 2- partículas não retidas a 35 mesh (partícula fina).

Após a moagem, determinou-se para todas as biomassas o teor de água em base úmida (TUbu\%), seguindo as recomendações da TAPPI T 257 om - 85/96 (Technical Association of Pulp and Paper, 1996).

A análise imediata da biomassa foi realizada com o intuito de caracterizar as amostras energeticamente. Foi avaliado o teor de material volátil, teor de cinzas e teor de carbono fixo, de acordo com a norma ASTM D 1762-84 (American Society for Testing Materials, 2007).

Para a determinação do poder calorífico superior (PCS, cal g ${ }^{-1}$ ou kcal kg-1), foi utilizado um calorímetro modelo IKA C2000 acoplado a um refrigerador IKA KV 600 em unidade de cal $\mathrm{g}^{-1}$, baseando-se na adaptação da norma ASTM E711-87 (American Society for Testing Materials, 2004) e na quantidade de $0,7 \mathrm{~g}$ de amostra por ensaio. As amostras utilizadas foram aquelas que após prévio peneiramento passaram pela abertura de 60 mesh. A determinação do PCS foi dada pela média da duplicata quando dois valores consecutivos foram inferiores a $40 \mathrm{cal} \mathrm{g}^{-1}$, conforme recomendação da norma utilizada. Também foi determinado o poder calorífico inferior (PCI, cal g-1 ou kcal kg-1). O PCI, em base seca, foi calculado pela fórmula de Launt (equação 1), citada por Gomide (1984).

$$
\mathrm{PCI}=\mathrm{PCS}-600[(9 \mathrm{H} / 100)]
$$

Onde: $\mathrm{PCI}=$ poder calorífico inferior $\left(\mathrm{kcal} \mathrm{kg}^{-1}\right)$; $\mathrm{PCS}=$ poder calorífico superior $\left(\mathrm{kcal} \mathrm{kg}^{-1}\right)$; e $\mathrm{H}=$ teor de hidrogênio (\% em base seca).

Utilizou-se como teor de hidrogênio de $6 \%$ para as seis biomassas estudadas, conforme observado na Tabela 1, exceto para a biomassa de pinhão manso, onde se adotou a média para os valores presentes na literatura citada $(6,54 \%)$.

Tabela 1. Valores citados na literatura para o teor de hidrogênio $(\%)$, referentes as seis biomassas estudadas.

\begin{tabular}{lcc}
\hline \multicolumn{1}{c}{ Biomassa } & $\begin{array}{c}\text { Teor de } \\
\text { hidrogênio (\%) }\end{array}$ & Fonte \\
\hline Pinus & 6 & Calegari et al. (2005) \\
Pinus & 6 & Brito \& Barrichelo (1982) \\
Tauari & 6 & Brito \& Barrichelo (1982) \\
\hline Cumaru & 6 & Brito \& Barrichelo (1982) \\
\hline Casca de arroz & 6,08 & Macêdo (2012) \\
\hline Bagaço de cana & 5,9 & Horst (2013) \\
\hline Bagaço de cana & 5,77 & Silva (2013) \\
\hline Bagaço de cana & 6,3 & Aboyade et al. (2011) \\
\hline Bagaço de cana & 5,87 & Macêdo (2012) \\
\hline Torta de pinhão manso & 6,92 & Labegalini (2013) \\
\hline Torta de pinhão manso & 6,7 & Kurniawan \& Ismadji \\
\hline Torta de pinhão manso & 6 & Ribeiro (2012) \\
\hline
\end{tabular}

Por fim, foi determinado o poder calorífico útil (PCU, cal g-1 ou kcal kg-1), levando-se em consideração o teor de água da biomassa (equação 2), calculado de acordo com as recomendações de Brito (1993) e Vale et al. (2007).

$$
\text { PCU }=\text { PCI }(1-\mathrm{Ubu})-600 \mathrm{Ubu}
$$

Onde: $\mathrm{PCU}=$ poder calorífico útil $\left(\mathrm{kcal} \mathrm{kg}^{-1}\right) ; \mathrm{PCI}=$ poder calorífico inferior $\left(\mathrm{kcal} \mathrm{kg}^{-1}\right)$; Ubu $=$ teor de umidade da amostra em base úmida.

Os briquetes foram confeccionados em prensa briquetadeira da marca Irmãos Lippel, modelo LB32 , onde se acoplou uma máquina sopradora para o resfriamento acelerado dos briquetes. 
Confeccionaram-se seis briquetes (corpo-de-prova) por tratamento, utilizando-se para cada um $40 \mathrm{~g}$ da amostra classificada e um teor de água próximo a 10\%, para partículas grossas e partículas finas, temperatura de compactação de 130 ou $200{ }^{\circ} \mathrm{C}\left( \pm 10{ }^{\circ} \mathrm{C}\right)$, pressões de compactação do manômetro de $80 \mathrm{ou} 120 \mathrm{kgf} \mathrm{cm}^{-2}$ e tempo de compactação de 5 min, contados a partir do momento em que se atingiu a pressão estabelecida. $\mathrm{O}$ tempo de compactação foi o mesmo citado por Quirino et al. (2012).

Desta forma, para cada biomassa, os parâmetros de densificação que influenciam a densidade energética dos briquetes resultaram no delineamento experimental, conforme apresentado na Tabela 2.

Tabela 2. Delineamento experimental para a avaliação dos parâmetros de briquetagem: granulometria, temperatura e pressão para cada biomassa agroflorestal estudada.

\begin{tabular}{cccc}
\hline Tratamento & Granulometria & Temperatura $\left({ }^{\circ} \mathbf{C}\right)$ & $\begin{array}{c}\text { Pressão (kgf } \\
\left.\mathbf{c m}^{-2}\right)\end{array}$ \\
\hline 1 & Grossa & 130 & 80 \\
2 & Grossa & 130 & 120 \\
3 & Grossa & 200 & 80 \\
4 & Grossa & 200 & 120 \\
\hline 5 & Fina & 130 & 80 \\
6 & Fina & 130 & 120 \\
7 & Fina & 200 & 80 \\
8 & Fina & 200 & 120 \\
\hline
\end{tabular}

Onde: as partículas grossas ficaram retidas em malha até 35 mesh; e partículas finas com malha superior a 35 mesh.

Todos os corpos-de-prova confeccionados foram dispostos em câmara climática com temperatura e umidade relativa controlada $\left(20{ }^{\circ} \mathrm{C}\right.$ e $65 \%$ de umidade relativa), por um período de aproximadamente 90 dias.

A densidade aparente (DA, $\mathrm{g} \mathrm{cm}^{-3}$ ) foi determinada pelo método estereométrico, no qual cada corpo-deprova teve a sua massa determinada em balança de sensibilidade igual a $10^{-2} \mathrm{~g}$ e teve suas dimensões determinadas por paquímetro com sensibilidade de $10^{-2} \mathrm{~mm}$. A densidade aparente foi calculada após permanecerem em câmara climática, pela relação entre massa e volume, considerando-se a forma cilíndrica dos corpos-de-prova.

A densidade energética (DE, Gcal $\mathrm{m}^{-3}$ ) foi calculada pelo produto entre o poder calorífico útil $(\mathrm{PCU})$ e a densidade aparente do briquete (DA).

A análise estatística foi realizada pelo software SPSS (Statistical Package for Social Sciences) v.
19 para sistema operacional Windows (IBM, 2010). Inicialmente, baseou-se em uma avaliação descritiva, considerando-se os valores máximos, mínimos, a média, o desvio padrão e o coeficiente de variação para o teor de carbono fixo, teor de materiais voláteis, teor de cinzas, densidade aparente unitária (DA, $\mathrm{g} \mathrm{cm}^{-3}$ ) e densidade energética (DE, Gcal $\mathrm{m}^{-3}$ ). Também foram analisadas as propriedades energéticas das biomassas, poder calorífico superior (PCS, $\mathrm{kcal} \mathrm{kg}^{-1}$ ), poder calorífico inferior (PCI, $\mathrm{kcal} \mathrm{kg}^{-1}$ ) e poder calorífico útil (PCU, $\mathrm{kcal} \mathrm{kg}^{-1}$ ).

Posteriormente, investigou-se o efeito dos parâmetros da briquetagem para cada biomassa por análise de variância (ANOVA), considerando-se como fonte de variação os parâmetros da briquetagem (granulometria, temperatura e pressão). As diferenças entre os grupos foram analisadas pelo teste Tukey a um nível de significância de $5 \%$, para cada variável.

\section{Resultados e discussão}

\section{Análise da biomassa}

Os resultados para a análise imediata das seis biomassas estudadas, considerando o teor de materiais voláteis (TMV\%), o teor de cinzas (TCZ\%) e o teor de carbono fixo (TCF\%), estão apresentados na Figura 1.

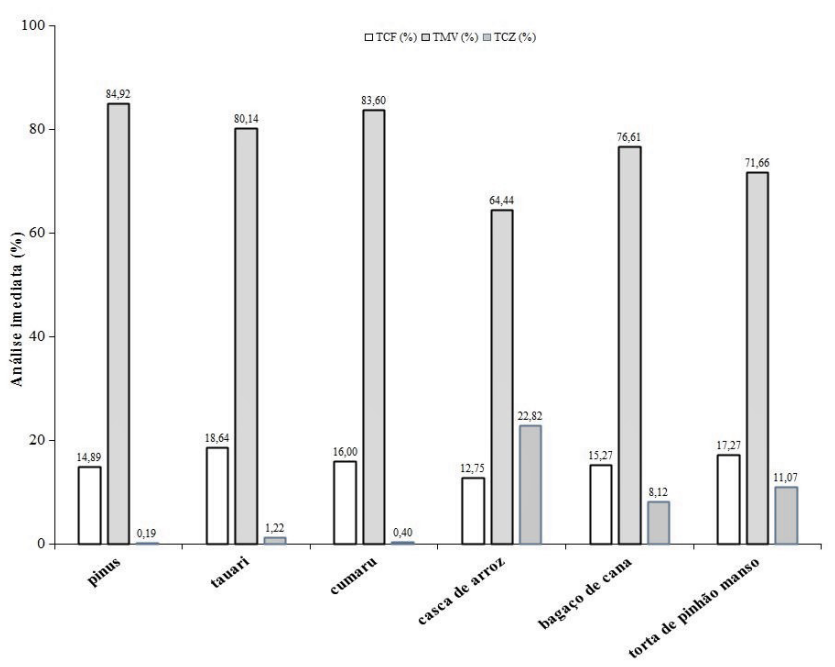

Figura 1. Valores do teor de carbono fixo, materiais voláteis e cinzas para as biomassas de pinus, tauari, cumaru, casca de arroz, bagaço de cana e torta de pinhão manso, onde: TCF $=$ teor de carbono fixo; TMV = teor de materiais voláteis; e $\mathrm{TCZ}=$ teor de cinzas.

Os maiores valores de conteúdo volátil foram observados para os resíduos florestais (média de 
$82,89 \%$ ), sendo aproximadamente $17 \%$ superior à média dos resíduos agrícolas $(70,90 \%)$, destacando o alto valor para a biomassa de pinus $(84,92 \%)$.

De acordo com McKendry (2002), o teor de materiais voláteis está relacionado à reatividade da biomassa à queima, ou seja, à facilidade de ignição da biomassa devido à volatilização de substâncias do material durante o aquecimento. Desta forma, destacou-se a maior reatividade das biomassas florestais.

Com relação às cinzas, os maiores valores observados foram para os resíduos agrícolas, sendo em média, $2.233 \%$ superiores aos resíduos florestais. Destaca-se que o efeito da adubação para elevar o potencial produtivo do solo e para atender as demandas nutricionais das culturas agrícolas, promoveu o acúmulo de minerais na constituição dos tecidos vegetais, resultando em teores mais altos de cinzas (Paula et al., 2011a; Vale et al., 2011). Em estudo para a caracterização da biomassa de pinhão manso, Vale et al. (2011), obtiveram teor de cinzas de aproximadamente $8 \%$, onde foram atribuídos, em parte, à presença significativa de macro e micronutrientes (ferro, zinco, cobre, potássio, cálcio, fósforo, sódio e magnésio).

O teor de cinzas mais alto foi observado na biomassa da casca de arroz, que ultrapassou $22 \%$. De acordo com Paula et al. (2011a), este alto valor de cinzas está ligado ao elevado conteúdo de sílica $\left(\mathrm{SiO}_{2}\right)$ presente nesta biomassa, que representa cerca de $8 \%$ do total das cinzas. Como efeito negativo, a grande quantidade de minerais nas cinzas pode reduzir o poder calorífico desta biomassa assim como interferir na condução de calor, por se tratar de um material com características de isolamento térmico (Furtado et al., 2010; Souza et al., 2012b).

Com relação ao teor de carbono fixo, os resíduos florestais apresentaram médias 9,34\% superior aos resíduos agrícolas, destacando o maior valor para a biomassa de tauari $(18,64 \%)$. Segundo Brito \& Barrichello (1982), a vantagem para maiores valores de carbono fixo se dá por uma liberação da energia presente na biomassa de uma forma mais lenta, implicando em maior tempo de residência nos fornos.

Os resultados de poder calorífico superior (PCS), poder calorífico inferior (PCI) e poder calorífico útil (PCU) estão apresentados na figura 2. O poder calorífico útil foi calculado em função do teor de umidade (base seca) das biomassas de pinus $(7,49 \%)$, tauari $(7,66 \%)$, cumaru $(7,83 \%)$, casca de arroz $(8,93 \%)$, bagaço de cana $(6,37 \%)$ e torta de pinhão manso $(11,03 \%)$.

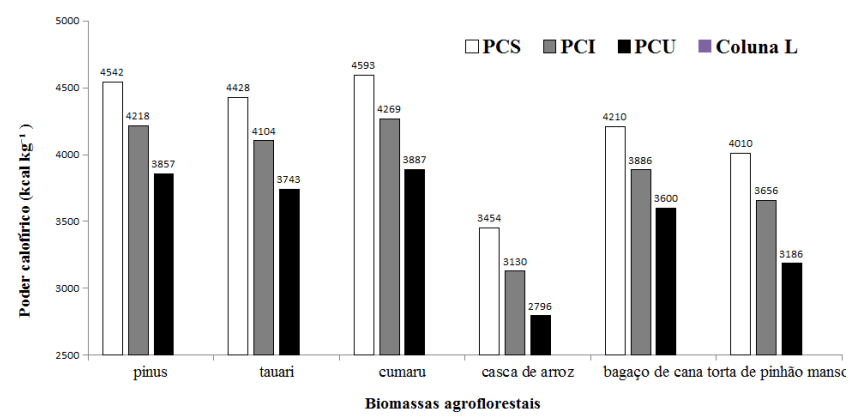

Figura 2. Valores do poder calorífico das seis biomassas agroflorestais estudadas, onde: $\mathrm{PCS}=$ poder calorífico superior; PCI = poder calorífico inferior; e PCU $=$ poder calorífico útil.

Observou-se que a média do poder calorífico superior para os resíduos florestais $\left(4.521 \mathrm{kcal} \mathrm{kg}^{-1}\right)$ foi cerca de $16 \%$ superior ao valor médio obtido para os resíduos agrícolas $\left(3.891 \mathrm{kcal} \mathrm{kg}^{-1}\right)$. Dentre os resíduos florestais, destacou-se o cumaru, com $4.593 \mathrm{kcal} \mathrm{kg}^{-1}$. Dentre os resíduos agrícolas, o bagaço de cana se mostrou com maior poder calorífico superior $\left(4.210 \mathrm{kcal} \mathrm{kg}^{-1}\right)$. De acordo com Quirino et al. (2004) o poder calorífico superior pouco varia entre as espécies florestais (média de $4.700 \mathrm{kcal} \mathrm{kg}^{-1}$ ), porém, são normalmente superiores aos resíduos agrícolas (média de $3.600 \mathrm{kcal} \mathrm{kg}^{-1}$ ) devido à constituição química das madeiras.

Os resultados obtidos para o PCS e PCI das biomassas estudadas foram compatíveis com os valores citados na literatura, conforme apresentado na Tabela 3.

Tabela 3. Valores citados na literatura para o conteúdo energético das seis biomassas estudadas.

\begin{tabular}{|c|c|c|c|}
\hline \multirow[t]{2}{*}{ Biomassa } & \multicolumn{2}{|c|}{$\begin{array}{c}\text { Conteúdo energético } \\
\left(\mathrm{kcal} \mathrm{kg}^{-1)}\right.\end{array}$} & \multirow[t]{2}{*}{ Fonte } \\
\hline & PCS & PCI & \\
\hline Pinus** & 4.786 & $4.462 *$ & Quirino et al. (2004) \\
\hline Tauari & 4.735 & $4.411 *$ & Quirino et al. (2004) \\
\hline Cumaru & 4866 & $4.542 *$ & Quirino et al. (2004) \\
\hline Casca de arroz & 3.812 & 3.445 & Paula et al. (2011b) \\
\hline Casca de arroz & 3.852 & $3.528^{*}$ & Jenkins (1990) \\
\hline Bagaço de cana & 4.274 & 3.855 & Paula et al. (2011a) \\
\hline Bagaço de cana & 4.139 & 3.815 & Jenkins (1990) \\
\hline Torta de pinhão manso & 5.122 & $4.798^{*}$ & Vale et al. (2011) \\
\hline
\end{tabular}

Onde: $\mathrm{PCS}=$ poder calorífico superior; $\mathrm{PCI}=$ poder calorífico inferior; $*$ valor calculado com base no teor de hidrogênio a 6\%; **espécie Pinus elliottii.

Com relação ao PCU mostrado na Figura 2, que leva em consideração a umidade presente no briquete, a média dos valores para os resíduos florestais (3.829 $\mathrm{kcal} \mathrm{kg}^{-1}$ ) foi superior em 19,88\% em relação à média dos resíduos agrícolas $\left(3.194 \mathrm{kcal} \mathrm{kg}^{-1}\right)$. 


\section{Análise dos briquetes}

Todos os briquetes confeccionados apresentaram estrutura íntegra e sem rachaduras, com exceção dos tratamentos 7 e $8 \mathrm{com}$ a torta de pinhão manso e o tratamento 8 com cumaru. Nestes dois casos, a produção dos briquetes foi inviabilizada, pois todos os briquetes formados apresentaram rachaduras profundas causadas por estouros após a liberação da pressão na máquina briquetadeira e esmagamentos causados pela contrapressão do pistão superior. Nestes tratamentos, variou-se apenas a pressão de 80 e $120 \mathrm{kgf} \mathrm{cm}^{-2}$, associada a $200{ }^{\circ} \mathrm{C}$ de temperatura e a partícula com granulometria não retida na peneira de 35 mesh.

Desta forma, acredita-se que a produção de briquetes foi inviabilizada pela grande formação de vapores de água e do óleo presente na torta de pinhão manso, não permitindo a agregação das partículas a fim de formar o briquete. Com relação aos briquetes de cumaru, possivelmente a liberação de extrativos e vapores de água promoveram os estouros e rachaduras, tornando esta biomassa nessa condição, não briquetável.

Na Tabela 4 são apresentados os resultados obtidos para a densidade aparente $\left(\mathrm{DA}-\mathrm{g} \mathrm{cm}^{-3}\right)$ dos briquetes confeccionados com as seis biomassas estudadas.

Tabela 4. Densidade aparente dos briquetes $\left(\mathrm{DA}-\mathrm{g} \mathrm{cm}^{-3}\right)$ com as seis biomassas estudadas em função dos oito tratamentos propostos.

\begin{tabular}{|c|c|c|c|c|c|c|c|c|}
\hline \multirow[b]{2}{*}{ Biomassa } & \multicolumn{8}{|c|}{ Tratamentos } \\
\hline & 1 & 2 & 3 & 4 & 5 & 6 & 7 & 8 \\
\hline Pinus & $1,088 \mathrm{~d}$ & $1,110 \mathrm{~d}$ & $1,202 \mathrm{a}$ & $1,190 \mathrm{a}$ & $1,107 \mathrm{~b}$ & $1,098 \mathrm{~b}$ & $1,197 \mathrm{a}$ & $1,197 \mathrm{a}$ \\
\hline Tauari & $1,142 \mathrm{~cd}$ & $1,152 \mathrm{c}$ & $1,218 \mathrm{~b}$ & $1,223 \mathrm{~b}$ & $1,095 \mathrm{e}$ & $1,132 \mathrm{~d}$ & 1,248 a & $1,255 \mathrm{a}$ \\
\hline Cumaru & $1,203 \mathrm{c}$ & $1,237 \mathrm{ab}$ & $1,238 \mathrm{ab}$ & $1,250 \mathrm{a}$ & $1,232 \mathrm{~b}$ & $1,228 \mathrm{~b}$ & $1,243 \mathrm{ab}$ & - \\
\hline Casca de arroz & $1,142 \mathrm{~d}$ & $1,242 \mathrm{c}$ & $1,247 \mathrm{bc}$ & $1,307 \mathrm{a}$ & $1,257 \mathrm{bc}$ & $1,263 \mathrm{~b}$ & $1,298 \mathrm{a}$ & $1,305 \mathrm{a}$ \\
\hline Bagaço de cana & $1,162 \mathrm{~d}$ & $1,163 \mathrm{~d}$ & 1,258 a & $1,262 \mathrm{a}$ & $1,188 \mathrm{c}$ & $1,217 \mathrm{~b}$ & $1,268 \mathrm{a}$ & $1,275 \mathrm{a}$ \\
\hline Torta de pinhão manso & $1,295 \mathrm{a}$ & 1,287 a & $1,215 \mathrm{~b}$ & $1,22 \mathrm{~b}$ & $1,115 \mathrm{~d}$ & $1,18 \mathrm{c}$ & - & - \\
\hline
\end{tabular}

Médias com letras diferentes para a mesma biomassa são diferentes estatisticamente pelo teste Tukey a 5\% de significância; - briquetes não confeccionados.

Os briquetes do presente estudo apresentaram densidade aparente superior a $1 \mathrm{~g} \mathrm{~cm}^{-3} \mathrm{e}$, em muitos casos, valores superiores a $1,2 \mathrm{~g} \mathrm{~cm}^{-3}$, correspondendo à densidade aparente citada na literatura (Protásio et al., 2011; Paula et al., 2011b; Quirino et al., 2012). Tecnicamente, densidade aparente maior é desejável, uma vez que existe uma relação diretamente proporcional entre o aumento da densidade aparente e a densidade energética do briquete.

Excetuando-se os briquetes confeccionados com a torta de pinhão manso, uma análise geral dos resultados mostrou que existe uma tendência de elevação da densidade aparente para os tratamentos 3 e 4 (partículas grossas), assim como para os tratamentos 7 e 8 (partículas finas) utilizando-se a temperatura de $200^{\circ} \mathrm{C}$. Desta forma, do ponto de vista técnico, sugerese para a confecção dos briquetes destas biomassas temperatura mais alta $\left(200{ }^{\circ} \mathrm{C}\right)$ e pressão mais baixa $\left(80 \mathrm{kgf} \mathrm{cm}^{-2}\right)$.
O efeito do tamanho da partícula na densidade aparente foi restrito a poucos tratamentos, porém foi significativamente favorável quando se utilizou partículas mais finas das biomassas de tauari e casca de arroz na confecção do briquete, elevando-se a densidade aparente. Este comportamento também foi descrito por Kaliyan \& Morey (2009), ou seja, quanto menor o tamanho da partícula melhor foi a compactação, aumentando-se a densidade aparente. Ainda de acordo com estes autores, as partículas com dimensões pequenas são interessantes pelo fato de abrangerem área maior de superfície e promoverem melhor interação.

Portanto, o efeito da temperatura na elevação da densidade aparente foi mais significativo quando comparado à elevação da pressão, devido à diferença relativamente pequena entre as pressões de compactação (80 e $\left.120 \mathrm{kgf} \mathrm{cm}^{-2}\right)$. De forma semelhante, Paula et al. (2011b) observaram que o aumento da temperatura de compactação aumentou a densidade aparente 
dos briquetes de pergaminho do grão do cafeeiro e, consequentemente, a sua resistência mecânica.

Para os briquetes confeccionados com a torta de pinhão manso, acredita-se em uma possível degradação térmica da biomassa, resultando em uma diminuição da densidade aparente.

Na Tabela 5 são apresentados os valores médios da densidade energética (DE, Gcal m${ }^{-3}$ ) dos briquetes com as seis diferentes de biomassas.

Tabela 5. Densidade energética dos briquetes (DE - Gcal $\left.\mathrm{m}^{-3}\right)$ com as seis biomassas estudadas em função dos oito tratamentos propostos.

\begin{tabular}{lccccccccc}
\hline & \multicolumn{7}{c}{ Tratamentos } \\
\cline { 2 - 9 } \multicolumn{1}{c}{ Biomassa } & 1 & 2 & 3 & 4 & 5 & 6 & 7 & 8 \\
\hline Pinus & $4,03 \mathrm{~b}$ & $4,11 \mathrm{~b}$ & $4,44 \mathrm{a}$ & $4,39 \mathrm{a}$ & $4,09 \mathrm{~b}$ & $4,05 \mathrm{~b}$ & $4,43 \mathrm{a}$ & $4,37 \mathrm{a}$ \\
Tauari & $4,11 \mathrm{~cd}$ & $4,13 \mathrm{c}$ & $4,38 \mathrm{~b}$ & $4,40 \mathrm{~b}$ & $3,93 \mathrm{e}$ & $4,06 \mathrm{~d}$ & $4,49 \mathrm{a}$ & $4,51 \mathrm{a}$ \\
Cumaru & $4,50 \mathrm{c}$ & $4,63 \mathrm{ab}$ & $4,63 \mathrm{ab}$ & $4,67 \mathrm{a}$ & $4,60 \mathrm{~b}$ & $4,60 \mathrm{~b}$ & $4,65 \mathrm{ab}$ & - \\
Casca de arroz & $3,11 \mathrm{~d}$ & $3,38 \mathrm{c}$ & $3,39 \mathrm{bc}$ & $3,56 \mathrm{a}$ & $3,42 \mathrm{bc}$ & $3,44 \mathrm{~b}$ & $3,54 \mathrm{a}$ & $3,55 \mathrm{a}$ \\
Bagaço de cana & $3,95 \mathrm{~d}$ & $3,95 \mathrm{~d}$ & $4,27 \mathrm{a}$ & $4,29 \mathrm{a}$ & $4,04 \mathrm{c}$ & $4,14 \mathrm{~b}$ & $4,32 \mathrm{a}$ & $4,33 \mathrm{a}$ \\
\hline Torta de pinhão manso & $4,14 \mathrm{a}$ & $4,11 \mathrm{a}$ & $3,88 \mathrm{~b}$ & $3,89 \mathrm{~b}$ & $3,69 \mathrm{c}$ & $3,74 \mathrm{c}$ & - & - \\
\hline
\end{tabular}

Médias com letras diferentes para a mesma biomassa são diferentes estatisticamente pelo teste Tukey a $5 \%$ de significância; ' briquetes não confeccionados.

Observou-se que o comportamento da densidade energética se mostrou semelhante ao comportamento da densidade aparente (tabela 5), onde, de uma forma geral, maiores valores de densidade aparente determinaram maiores valores da densidade energética. Este fato foi observado tendo em vista uma relação diretamente proporcional entre estas variáveis e a condição semelhante de umidade após a climatização, proporcionando pouca variação no PCU.

Portanto, para a obtenção de maiores valores da densidade energética, a utilização da temperatura mais alta foi mais eficiente que a elevação da pressão de compactação e da redução do tamanho da partícula nos briquetes com pinus, tauari, casca de arroz e bagaço de cana. Por outro lado, nos briquetes com cumaru a variação dos parâmetros de briquetagem pouco influenciou a densidade energética. Para os briquetes com torta de pinhão manso, houve um decréscimo da densidade energética a partir do aumento da temperatura, possivelmente por uma leve degradação térmica da biomassa.

Desta forma, dentre os resíduos florestais, a DE mais elevada foi observada em briquetes de cumaru, chegando a 4,67 Gcal $\mathrm{m}^{-3}$ e em briquetes de tauari, com cerca de $4,51 \mathrm{Gcal} \mathrm{m}^{-3}$. Por outro lado, com resíduos agrícolas, em briquetes com bagaço de cana obteve-se 4,33 Gcal $\mathrm{m}^{-3}$ e com torta de pinhão manso, $4,14 \mathrm{Gcal} \mathrm{m}^{-3}$.

Protásio et al. (2011) determinaram a densidade energética de briquetes utilizando a densidade aparente, a aproximadamente $6 \%$ de umidade, e o poder calorífico superior, encontrando 5,69 $\mathrm{Gcal} \mathrm{m}^{-3}$ para os briquetes da casca de café, 4,22 Gcal m${ }^{-3}$ para os de resíduos de milho e 4,17 Gcal $\mathrm{m}^{-3}$ para briquetes da serragem de eucalipto. Furtado et al. (2010) também utilizaram o poder calorífico superior obtido em condição seca e a densidade aparente a cerca de $9 \%$ de teor de umidade para o cálculo densidade energética de briquetes com casca, cavacos e serragem de pinus. Para os briquetes confeccionados com a casca de pinus e utilizando-se $120{ }^{\circ} \mathrm{C}$ e pressão de $132,5 \mathrm{kgf} \mathrm{cm}^{-2}$, observaram 5,4 Gcal $\mathrm{m}^{-3}$. O valor final nas condições de cálculo da densidade energética nos dois casos possivelmente estaria superestimado, pois não se desconsiderou o teor de umidade do briquete, dificultando assim a comparação com os resultados deste trabalho.

Sendo assim, acredita-se que a metodologia para se calcular a densidade energética de briquetes que mais se aproxima de uma aplicação prática seria a multiplicação do poder calorífico útil e a densidade aparente do briquete, com o objetivo de não superestimar o valor desta propriedade. 


\section{Conclusões}

As biomassas florestais apresentaram as propriedades energéticas (poder calorífico superior, inferior e útil e teores de carbono fixo, de materiais voláteis e de cinzas) mais favoráveis à briquetagem e à combustão.

A elevação da temperatura de compactação foi mais eficiente na melhoria da qualidade dos briquetes, proporcionando maiores valores de densidade aparente e de densidade energética, na maior parte dos tratamentos. No entanto, a variação na pressão de compactação pouco influenciou nas propriedades finais do briquete. Recomenda-se a aplicação da pressão de $80 \mathrm{kgf} \mathrm{cm}^{-2}$ para o processo de briquetagem.

$\mathrm{O}$ efeito da redução no tamanho das partículas foi mais evidente nos briquetes de tauari e casca de arroz, promovendo elevação da densidade aparente e energética.

\section{Referências}

Aboyade, A. O et al. Non-isothermal kinetic analysis of the devolatilization of corn cobs and sugar cane bagasse in an inert atmosphere. Thermochimica Acta, v. 517, n. 1-2, p. 81-89, 2011. DOI: 10.1016/j.tca.2011.01.035.

American Society for Testing Materials. ASTM D1762-84: standard test method for chemical analysis of wood charcoal. Philadelphia, 2007. 2 p.

American Society for Testing Materials. ASTM E711-87: standard test method for gross calorific value of refuse-derived fuel by the bomb calorimeter. Philadelphia, 2004. 8 p.

Brand, M. A. et al. Influência da pressão e material nas propriedades de briquetes de biomassa florestal. In: CONGRESSO BRASILEIRO SOBRE FLORESTAS ENERGÉTICAS, 1., 2009, Belo Horizonte. Anais... Colombo: Embrapa Florestas, 2009. (Embrapa Florestas. Documentos, 178).

Brito, J. O. Expressão da produção florestal em unidades energéticas, In: CONGRESSO FLORESTAL PANAMERICANO, 1.; CONGRESSO FLORESTAL BRASILEIRO, 7., 1993, Curitiba. Floresta para o desenvolvimento: política, ambiente, tecnologia e mercado: anais. São Paulo: SBS; [S.1.]: SBEF, 1993. p. 280-282.

Brito, J. O. \& Barrichelo, L. E. G. Aspectos técnicos da utilização da madeira e carvão vegetal como combustíveis. In: SEMINÁRIO DE ABASTECIMENTO ENERGÉTICO INDUSTRIAL COM RECURSOS FLORESTAIS, 2., 1982, São Paulo. Palestras apresentadas. São Paulo: Secretaria da Indústria, Comércio, Ciência e Tecnologia, 1982. p. 101-137.

Calegari, L. et al. Características de algumas biomassas usadas na geração de energia no Sul do Brasil. Biomassa \& Energia, v. 2, n. 1, p. 37-46, 2005 .
Couto, L. C. \& Barcellos, D. C. As relações água-madeira e sua influência nas propriedades físicas. Biomassa \& Energia, v. 4, n. 2, p. 173-190, 2011.

Furtado, T. S. et al. Variáveis do processo de briquetagem e qualidade de briquetes de biomassa florestal. Pesquisa Florestal Brasileira, v. 30, n. 62, p. 101-106, 2010. DOI: 10.4336/2010.pfb.30.62.101.

Gomide, R. Estequiometria industrial. 3. ed. São Paulo: Cenpro, 1984. 423 p.

Horst, D. Avaliação da produção energética a partir de ligninas contidas em biomassas. 2013. 106 f. Dissertação (Mestrado em Engenharia de Produção) - Universidade Tecnológica Federal do Paraná, Ponta Grossa.

IBM. Released 2010: IBM SPSS Statistics for Windows, Version 19.0. Armonk, NY: 2010.

Jenkins, B. M. Fuel properties for biomass materials. In: INTERNATIONAL SYMPOSIUM ON APPLICATION AN MANAGEMENT OF ENERGY IN AGRICULTURE: THE ROLE BIOMASS FUELS, 1., 1990, New Delhi. Proceedings... Ludhiana: Punjab Agricultural University, 1990. p. 21-23.

Kaliyan, N. \& Morey, R. V. Factors affecting strength and durability of densified biomass products. Biomass and Bioenergy, v. 33, n. 3, p. 337-359, 2009.

Karunanithy, C. et al. Physiochemical characterization of briquettes made from different feedstocks. Biotechnology Research International, v. 2012, ID 165202, p. 1-12, 2012. DOI: $10.1155 / 2012 / 165202$.

Kurniawan, A. \& Ismadji, S. Potential utilization of Jatropha curcas L. press cake residue as new precursor for activated carbon preparation: Application in methylene blue removal from aqueous solution. Journal of the Taiwan Institute of Chemical Engineers, v. 42, n. 5, p. 826-836, 2011. DOI: 10.1016/j.jtice.2011.03.001.

Labegalini, A. Obtenção de biochar a partir da pirólise rápida da torta de pinhão manso: uso como adsorvente e suporte. 2013. 116 f. Dissertação (Mestrado em Agroquímica) - Instituto de Química Ambiental, Universidade Federal de Lavras, Lavras.

Macêdo, L. A. Influência da composição da biomassa no rendimento em condensáveis do processo de torrefação. 2012. 49 f. Dissertação (Mestrado em Ciências Florestais) - Universidade de Brasília, Brasília, DF.

McKendry, P. Energy production from biomass (part 1): overview of biomass. Bioresource Technology, n. 83, p. 37-46, 2002. DOI: 10.1016/S0960-8524(01)00118-3.

Melo, R. R. et al. Variação radial e longitudinal da densidade básica da madeira de Pinus elliottii Engelm. com diferentes idades. Ciência da Madeira, v. 4, n. 1, p. 83-92, 2013.

Olakeji, J. T. \& Enweremadu, C. C. The effects of some processing parameters on physical and densification characteristics of corncob briquettes. International Journal of Energy Engineering, n. 2, v. 1, p. 22-27, 2012.

Pinheiro, G. F. et al. Densidade energética de resíduos vegetais. Biomassa \& Energia, v. 2, n. 2, p. 113-123, 2005. 
Protásio, T. P. et al. Compactação de biomassa vegetal visando à produção de biocombustíveis sólidos. Pesquisa Florestal Brasileira, v. 31, n. 68, p. 273-283, 2011. DOI: 10.4336/2011.pfb.31.68.273.

Quirino, W. F. \& Brito, J. O. Características e índice de combustão de briquetes de carvão vegetal. Brasília, DF: IBAMA, Laboratório de Produtos Florestais, 1991. (Série técnica, n. 13).

Quirino, W. F. et al. Densitometria de raios x na análise da qualidade de briquetes de resíduos de madeira. Scientia Forestalis, v. 40, n. 96, p. 525-536, 2012.

Quirino, W. F. et al. Poder calorífico da madeira e de resíduos lignocelulósicos. Biomassa \& Energia, v. 1, n. 2, p. 173-182, 2004.

Paula, L. E. e R. et al. Characterization of residues from plant biomass for use in energy generation. Cerne, v. 17, n. 2, p. 237-246. 2011a. DOI: $10.1590 / \mathrm{S} 0104-77602011000200012$

Paula, L. E. e R. et al. Produção e avaliação de briquetes de resíduos lignocelulósicos. Pesquisa Florestal Brasileira, v. 31, n. 66, p. $103-$ 112, 2011b. DOI: 10.4336/2011.pfb.31.66.103.

Ribeiro, R. F. L. Avaliação de tortas de oleaginosas com potencial para produção de biodiesel na obtenção de materiais adsorventes para remoção de metais em meio aquoso. 2012. 159 f. Tese (Doutorado em Química) - Universidade Federal de Minas Gerais, Belo Horizonte.
Silva, C. V. Caracterização do bio-óleo produzido por pirólise rápida do bagaço de cana de açúcar. 2013. 90 f. Dissertação (Mestrado em Química) - Instituto de Química, Universidade Federal de Uberlândia, Uberlândia.

Souza, M. M. et al. Estimativa de poder calorífico e caracterização para uso energético de resíduos da colheita e do processamento de Pinus taeda. Floresta, v. 42, n. 2, p. 325-334, 2012a. DOI: 10.5380/ rf.v42i2.26593.

Souza, S. N. M. et al. Potencial de geração de bioeletricidade com casca de arroz no Brasil. Cultivando o Saber, v. 5, n. 1, p. 1-8, 2012 b.

Technical Association of Pulp and Paper. Sampling and preparation wood for analysis: T $257 \mathrm{om}$ - 88. Atlanta, 1996.

Vale, A. T. et al. Caracterização energética e rendimento da carbonização de resíduos de grãos de café (Coffea arabica, $\mathrm{L})$ e de madeira (Cedrelinga catenaeformis, Duke). Cerne, n. 13, p. 416420, 2007.

Vale, A. T. et al. Potencial energético da biomassa e carvão vegetal do epicarpo e da torta de pinhão manso (Jatropha curcas). Cerne, v. 17, n. 2, p. 267-273, 2011. DOI: 10.1590/S0104-77602011000200015. 
